\title{
La formación por competencias en educación superior ${ }^{1}$
}

\author{
Competence-based training in higher education
}

\author{
Martha Janet Velasco Forero ${ }^{2}$
}

Para citar este artículo: Velasco Forero, M. J. (2019). La formación por competencias en educación superior. Revista ObIES, 3, 44-63.

Recibido: 31-octubre-2019 / Aceptado: 19-noviembre-2019

\section{Resumen}

Para adentrarse en temas como la internacionalización del currículo, desde la posibilidad de pasar de la disciplina a la interdisciplinariedad, en la cual sea posible recocer ámbitos culturales y sociales diversos, se hace necesario construir el concepto histórico de la formación por competencia en la educación superior, particularmente en América Latina y Colombia, así como explorar su complejidad con sus multiplicidades. Esto puede dar cuenta de un extenso y excesivo tratamiento, relacionado directamente con: la formación, los perfiles profesionales y laborales, el favorecimiento de la empleabilidad, el currículo, las prácticas docentes, los sistemas evaluativos, la psicología del trabajo, entre otros rasgos distintivos de orden conceptual. Se reconoce que las nuevas nociones relacionadas con el currículo por competencias expresan un constructo mental que, desde el punto de vista metodológico, debe ser predecible, medible, constatable, además de deseado.

Palabras clave: competencias; formación por competencias; currículo; currículo por competencias.

\begin{abstract}
In order to explore issues such as the internationalization of the curriculum, from the possibility of moving from one discipline to another, in which it is possible to recognize diverse cultural and social areas, it is necessary to construct the historical concept of competency-based training in higher education, particularly in Latin America and Colombia, as well as to explore its complexity with its multiplicities. This may give rise to an extensive and excessive treatment, directly related to: training, professional and work profiles, the promotion of employability, the curriculum, teaching practices, assessment systems, and work psychology, among other distinctive features of a conceptual nature. It is recognized that the new notions related to the competency-based curriculum express a mental construct that, from a methodological point of view, should be predictable, measurable, verifiable, and desired.
\end{abstract}

Keywords: competencies; competency-based training; curriculum; competency-based curriculum.

1 El presente artículo hace parte de la reflexión teórica derivada de la investigación realizada en el marco de la tesis doctoral "Currículo y gobernanza en educación superior: una mirada desde las competencias" del doctorado en Educación Superior de la Universidad de Palermo/ Argentina, finalizada en el año 2018.

2 Ph.D en Educación Superior por la Universidad de Palermo (Argentina). Actualmente se desempeña como docente en la Universidad Distrital Francisco José de Caldas (Bogotá, Colombia). Correo electrónico: marthavelasco16@gmail.com 


\section{El enfoque de competencias en educación superior}

El término competencias ${ }^{3}$ es un concepto plurisignificativo y en permanente evolución. Al mismo tiempo, "resulta ser un término confuso, ambiguo y polisémico, sujeto a diversas interpretaciones y significados, lo que dificulta enormemente su utilización en la docencia y en la evaluación" (Gómez, s. f., p. 1). De igual manera, tal como expone Díaz Barriga:

[...] el concepto de competencia es polisémico. En los discursos y reformas educativas actuales el manejo del término es a veces indiscriminado, pues en muchas ocasiones no queda claro cuál es el referente teórico-conceptual y los límites referenciales desde donde se está planteando el sentido del término. (Diaz-Barriga, 2006, p. 12)

A pesar de su multiplicidad semántica ${ }^{4}$, han sido numerosos los estudios dedicados a dar cuenta del origen del concepto de competencia entre los cuales vale la pena destacar los trabajos de Dalziel, Cubeiro y Fernández (1996); Levy-Leboyer (1997); Mertens (1997); Mitrani, Dalziel y Suárez de Puga (1992); Spencer Jr. y Spencer (1993); y Voorhees (2002). Investigaciones que abordan minuciosamente la aparición del término de competencia en el campo de la educación llegan al consenso de considerar que fue David McClelland (1973) quien formalizó la enunciación del término competence desde el campo de la psicología, cuando en el año de $1973^{5}$ sus trabajos en la Universidad de Harvard asumen una visión crítica frente a conocimientos,

3 Etimológicamente, los conceptos de competencia y competente provienen del latín competentia y competens, entis, respectivamente.

4 Autores como Hoffman (1999), Wood y Payne (1998), Gimeno Sacristán y Pérez Gómez (2009), entre otros, plantean que "no existe consenso respecto a su significación" y sus distintos usos, y ubican las competencias como un "fenómeno contradictorio con consecuencias contradictorias" (Gimeno Sacristán et al., 2009, p. 11).

5 Algunos teóricos mantienen en discusión la aparición conceptual del término competencia; para algunos sería la década de 1960, para otros el concepto se introduce hacia 1950; incluso, existe quienes consideran que es hacia finales de 1920. aptitudes y actitudes que son considerados como copredictores suficientes del desempeño laboral. Análisis que, según Blas Aritio (2007), posibilitó inferir distintas variables para el estudio de las competencias. De otro lado, algunos teóricos de la educación circunscriben y atribuyen el término de competencias a la aparición de algunos movimientos culturales y sociales florecidos en la sociedad norteamericana ${ }^{6}$ de las décadas de 1960 y 1970.

No obstante, frente a la complejidad del término las investigaciones adelantadas por García San Pedro (2010) ponen en evidencia cómo en la teoría el concepto de competencia se encuentra matizado por los autores que han abordado el tema como objeto de estudio y sus respectivas nacionalidades de origen. Así, entre las denominaciones halladas por García San Pedro, se pueden señalar:

[...] "Generic Graduate Attributes" (Barrie, 2005; 2006), "Generic Capabilities" (Bowden et al., 2000), "Graduate Attributes Movement" (Chanock, 2004), "Graduate Skills" (Chanock, Clerehan y Moore, 2004), "Personal Transferable Skills" (Drummond, Nixon y Wiltshire, 1998), "Graduate Capability Development" (Kift, 2002). (2010, p. 40)

De ahí que sus multiplicidades dan cuenta de un extenso y excesivo tratamiento, relacionado directamente con la formación, los perfiles profesionales y laborales, el favorecimiento de la empleabilidad, el currículo, las prácticas docentes, los sistemas evaluativos, la psicología del trabajo ${ }^{7}$, entre otros rasgos distintivos de orden conceptual.

6 En este sentido, Guillermet Fernández y Carina Rubau (2012) evidencian cómo a partir de la década de 1980 el concepto de competencia es utilizado en los países anglosajones como Inglaterra y Estados Unidos, así como en algunas naciones europeas tales como Francia y Alemania, con el propósito de seleccionar, capacitar y evaluar a los trabajadores, estableciéndose así una vinculación estrecha entre la capacitación o formación profesional, de los sujetos y el mundo del trabajo (Mastache, 2009).

7 Sobre esta concepción, para Díaz-Barriga (2011) desde la psicología del trabajo "se está incorporando esta noción para la educación en el mundo global". En otro sentido "la mayor parte de la literatura se dedica a temas relacionados con proponer estrategias para elaborar planes o programas de estudio, desatendiendo o desconociendo la problemática conceptual que subyace en este tema" (Díaz Barriga, 2006, p. 8). 
Ahora bien, en el desarrollo teórico contemporáneo el concepto de competencia ha tenido una gran expansión en la educación y la pedagogía. En este campo se asume el concepto desde los distintos niveles de escolaridad y se dedica un apartado importante a los procesos educativos de educación superior. Desde la educación técnica, tecnológica o p1rofesional se insiste en el desarrollo de competencias que posibiliten la formación profesional. Pues el término de competencias se encuentra inmerso y relacionado con el mundo laboral; por tanto, gran parte de su comprensión actual circula alrededor de la lógica de producción económica, fundamentalmente capitalista, el marco de la globalización económica internacional que se caracteriza por un alto nivel de fluidez y cambio, y que también demanda de los trabajadores mayores exigencias en cuanto a las habilidades individuales y colectivas para desempeñarse laboralmente.

Sobre esta lógica los trabajos de Fernández Guillermet y Rubau (2012) centran su atención en las denominadas competencias laborales y logran identificar tres momentos estelares en la evolución del concepto. El primero ve la competencia en términos de los desempeños involucrados en una serie de acciones específicas que el trabajador debe llevar a cabo. El segundo entiende la competencia como un conjunto de atributos del sujeto que incluye, entre otros, motivaciones, habilidades, conocimientos, representaciones de sí mismo y de la sociedad. Y el tercero, nombrado momento holístico, concibe la competencia como una combinación de atributos del sujeto y de las tareas a desempeñar en un contexto dado de trabajo (Gonczi, 1996; Vargas et al. 2001, citado por Mastache (2009).

Sin embargo, en esta dinámica las competencias instituyen un puesto esencial de referencia que determina las exigencias educativas: aptitudes, conocimientos y valores, de los programas académicos de educación superior en su transición al mercado laboral, en el marco de la sociedad de la información y del conocimiento.
En este marco, las competencias no solo hacen referencia al conjunto de conocimientos que posee una persona para desempeñar un trabajo, sino también a la capacidad de enfrentar problemas de orden laboral y a la capacidad de integrarse y adaptarse, de modo flexible, ante esas situaciones problémicas para poder plantear soluciones a dificultades que hoy no se conocen. La flexibilidad y la adaptación al devenir y las transformaciones del futuro, incluso a lo inesperado, es una característica importante de la teoría del modelo de competencias, pues:

[...] vivimos en una sociedad cambiante. Las competencias de hoy no son las de mañana. Los profesionales competentes no sólo deben poder sobrellevar los cambios, sino que deben ser capaces de participar en ellos activamente. [...] No se trata simplemente de poseer 'competencias', sino también de tener la capacidad para conversar acerca de ellas, para evaluarlas, para adquirir otras nuevas y descartar las viejas. (Barnett, 2001, p. 111)

Desde el punto de vista epistemológico son variadas las preocupaciones por analizar en el ámbito educativo las competencias expresadas por autores como: Barnett (2001), Perrenoud (2005), Mastache (2009) y Gimeno Sacristán (2009).

Es posible considerar varias posturas de escuelas o corrientes de pensamiento respecto a las competencias, para desde dichas comprender sus desarrollos y rasgos distintivos que caracterizan el sentido atribuido al término. Algunos ejemplos se pueden encontrar en las investigaciones de Martin Mulder, Tanja Weigel, Kate Collings, en los cuales es posible identificar tres enfoques de la competencia basados en aportes teóricos realizados por Norris (1991), Eraut (1994) y Wesselink, Biemans y Van den Elsen (2005), que se pueden condensar de la siguiente manera:

a. El enfoque conductista o behaviorista, promovido por McClelland (1973), quien usó el concepto de competencia, en vez del concepto de inteligencia, 
basado en la demostración, la observación y la evaluación de los comportamientos o conductas, y la definió como aquel conjunto de características de una persona que están relacionadas con el desempeño efectivo de un trabajo y pueden ser comunes en otras situaciones (Delamare Le Deist y Winterton, 2005; Spencer y Spencer, 1993; Gonczi 1994; McClelland, 1998). En este sentido, son adquiridas por medio de la formación y el desarrollo, se cimientan en la descripción de conductas observables o desempeños in situ.

b. En el enfoque genérico la competencia está más relacionada "con un desempeño global que sea apropiado a un contexto ${ }^{8}$ particular" (Hager, 2011, p. 533). Por ello, parte de identificar a las personas más efectivas y a sus características más distintivas (Norris, 1991) con el fin de identificar habilidades comunes que explican las variaciones en los distintos desempeños y que se pueden aplicar a diferentes grupos de profesionales. Hager (1998) por su parte subraya la influencia de los cambios en los contextos laborales. Se destacan investigaciones en torno a la personalidad (Barrick y Mount, 1991) para explicar las variaciones en el desempeño de un trabajo y las habilidades genéricas y básicas en el área de desarrollo curricular mencionadas por Mulder (1989), Nijhof y Mulder (1989) y Mulder y Thijsen (1990). El enfoque cognitivo está centrado en competencias generales cognitivas que incluyen modelos psicométricos sobre la inteligencia humana, modelos de procesamiento de la información y el modelo piagetiano de desarrollo cognitivo. Gira en torno a las competencias cognitivas especializadas, las cuales actúan como un grupo de prerrequisitos cognitivos que los individuos deben poseer para actuar bien en un área determinada. Otra interpretación del enfoque cognitivo es la diferenciación entre competencia y desempeño basado en la teoría de Chomsky (1980) sobre las reglas en las

8 Gonczi et al. (1995) confirmaron las diversas formas que las competencias genéricas pueden adoptar dependiendo de los contextos o de los lugares de trabajo. que se basa el aprendizaje y el uso del lenguaje necesarias para el desempeño lingüístico.

Autores como Mertens (1998), Rodríguez (2004) y Rodríguez Uribe (2009) consideran al humanismo como una corriente epistemológica en la que la esencia de ser competente consiste en ser ético, pues la convivencia en la solidaridad y la justicia son características importantes. Estos autores identifican tres enfoques y proponen una clasificación de acuerdo con los desarrollos teóricos y pedagógicos que han trabajado las competencias, a saber: el conductista, el funcionalista, el constructivista y el complejo, además de las taxonomías existentes en competencias básicas, genéricas y especializadas.

a. El enfoque conductista ${ }^{9}$ : basado en fundamentos positivistas, considera las competencias como características de las personas expresadas en comportamientos, causalmente relacionadas con un desempeño superior en un cargo o función de trabajo, por ello, enfatiza en comportamientos clave de las personas para la competitividad de las organizaciones. De ahí que centre su atención en las personas competentes al analizar los comportamientos sociales del alto rendimiento e identificando las competencias con rasgos aplicados al buen desempeño: lo que se debe ser.

c. El enfoque funcional: basado en el funcionalismo psicológico, considera la vida mental y el comportamiento humano en términos de adaptación activa al ambiente por parte de la persona; asume la competencia como la capacidad para realizar actividades y lograr resultados en una función productiva determinada, según criterios de desempeño. Por tanto, enfoca sus esfuerzos a la formación y capacitación en función de las necesidades del mercado laboral, privilegiando la descripción de productos y resultados frente a la descripción de procesos. De ahí que haga

9 Su principal representante es McClelland (1973) y es el modelo seguido por los Estados Unidos. Este modelo se basa en la teoría del comportamiento o de las conductas de los individuos (Watson, 1913). 
énfasis en el conjunto de atributos que deben tener las personas para cumplir con los propósitos de los procesos laborales y profesionales, enmarcados en funciones definidas; es decir, se concentra en lo que se debe hacer.

d. El enfoque constructivista: tiene como soporte epistemológico la teoría constructivista que valora positivamente las habilidades, conocimientos y destrezas para resolver dificultades en los procesos laborales-profesionales. Adicionalmente, se fundamenta desde el marco organizacional de la teoría de Schwartz (1995). En este enfoque la competencia se encuentra relacionada con cada una de las actividades que vocacional o laboralmente hacen merecer el apelativo de experto, basada en un proceso de construcción de representaciones mentales por niveles de complejidad crecientes. La competencia se construye no solo de la función que nace del mercado, sino que concede igual importancia a la persona, a sus objetivos y posibilidades. Por ello, parte del análisis de la relación entre la persona, el grupo y su entorno, así como entre la formación y el empleo, las expectativas, los conocimientos y las experiencias son un insumo indispensable.

e. El enfoque complejo: basado en el pensamiento complejo, considerado como el método de construcción del conocimiento que se basa en el tejido de relaciones entre las partes y el todo, y que es considerado desde una realidad holística y sistemática. Tobón (2008) pone especial énfasis en los sentidos de apropiar las competencias como procesos complejos de desempeño ante acciones y problemas con aptitud y ética, escudriñando la construcción personal, la calidad de vida y el desarrollo social y económico sostenible y en equilibro con el ambiente. El paradigma de la complejidad provee de una epistemología que posibilita una construcción de saberes que tiene en cuenta el entretejido de las partes, las relaciones, el caos y la incertidumbre.
En otra sistematización, Díaz-Barriga (2011) propone una clasificación que parte de la identificación de algunas escuelas o corrientes de pensamiento e identifica los enfoques: laboral, funcional, etimológico, psicológicos conductual o socio-constructivista y el pedagógico didáctico.

a. El enfoque laboral: cuyo término llega a la educación desde el mundo del trabajo y es tomado como el resultado de un análisis de tareas ampliamente desarrollado para definir los perfiles de un puesto, lo que resulta altamente conveniente.

f. El funcional o sistémico: defiende el aprendizaje con utilidad inmediata en la vida, así, simultáneamente critica la perspectiva escolar que reivindica el valor de los contenidos académicos por sí mismos, que ocasiona que estos tengan en el aula un tratamiento solo escolar o enciclopédico y, al mismo tiempo, reclama la necesidad de articular lo que se aprende en la escuela con la vida cotidiana.

g. El enfoque conductual o psicológico: contiene una serie de elementos comportamentales y se encuentra estrechamente ligado a la perspectiva laboral; tiene gran similitud con la teoría de objetivos comportamentales.

h. El enfoque etimológico: enmarca el término en la historia del desarrollo de la humanidad, justificando que no solo se refiere a su uso más común, el sentido que tiene una competencia deportiva, una social o profesional, sino acudiendo a su sentido etimológico y que, según el autor, refleja una perspectiva pre-foucaultniana que está ampliamente superada en el ámbito de las Ciencias Sociales (Díaz-Barriga, 2011).

i. El enfoque socialconstructivista: asume tres tendencias claras: la primera se refiere a reconocer el papel del sujeto en la construcción de su conocimiento; la segunda guarda estrecha relación con lo que denominan aprendizaje situado/ aprendizaje en contexto; mientras que la tercera se refiere al reconocimiento de la necesidad de 
graduar, de acuerdo con la complejidad intrínseca de la construcción del conocimiento, cada proceso de aprendizaje. Finalmente, el enfoque pedagógico-didáctico reconoce el debate didáctico detrás de la historia de las competencias y asume preguntas orientadoras frente a la discusión de cómo organizar y gestionar el aprendizaje (Díaz-Barriga, 2011).

De otro lado, Mertens (1997), citado por Zayas Agüero (2010), identifica la existencia de tres grandes tendencias en el estudio de las competencias:

a. El análisis conductista: que no se refiere a la corriente psicológica de esta misma denominación, sino al énfasis que se hace al papel de las características del individuo, es decir, parte del estudio de las personas que desempeñan bien su trabajo.

j. El enfoque funcional: que tiene un carácter evidentemente pragmático y conductista desde el punto de vista psicológico.

k. El análisis constructivista: que señala el carácter emergente de las competencias al tener como base el proceso de elaboración de las mismas por parte del investigador.

Según Boon y Van der Klink (2002), la competencia es un concepto confuso y útil que Ilena el vacío existente entre la educación y los requerimientos de trabajo, distinguiendo dos perspectivas: la geográfica y la psicológica. Para Tardif (2006), citado por Díaz-Barriga "existen al menos dos concepciones de competencias, una de corte conductual cercana a las propuestas de orden laboral y otra de corte sistémico que se ha anclado en las perspectivas cognitivas" (2011, p. 115).

Algunas nociones entrelazan la idea de lo práctico y vinculan la competencia directamente con el desempeño profesional, así, se encuentra a Colunga Santos y otros (2009), quienes afirman que hay un concepto centrado en el perfil y relacionado con la excelencia profesional, en el cual las competencias son comprendidas con un mayor alcance, ya que ser un profesional competente significa asumir una visión más integral en el proceso de búsqueda de un egresado capaz de dar respuesta eficiente a la diversidad de problemas y exigencias que el ejercicio de su profesión pueda plantearle. Consideran también el concepto de competencia de naturaleza holística y compleja, en el que se toman en consideración los elementos relativos a la tarea, a la excelencia profesional; además, se piensa en un entorno más complejo y global en el marco del cual se despliega la actividad profesional y en un conjunto de atributos personales imprescindibles para el ser y hacer competente.

Para Rueda Serón y otros (1997), las competencias son las capacidades para realizar funciones y situaciones de trabajo a los niveles requeridos en el empleo, incluyen la anticipación de problemas, la evaluación de las consecuencias del trabajo y la facultad de participar activamente en la mejora de la producción. Noción que se vincula al campo laboral al igual que las propuestas de Guerrero (1999) y Agudelo (2002). El primero la entiende como la capacidad de aplicar, en condiciones operativas y conforme al nivel requerido, las destrezas, conocimientos y actitudes adquiridas por la formación y la experiencia profesional, al realizar las actividades de una ocupación, incluidas las posibles nuevas situaciones que puedan surgir en el área profesional y ocupaciones afines. El segundo concibe la competencia como la disposición de conocimientos, habilidades, aptitudes y actitudes necesarios para desempeñarse eficazmente en situaciones específicas de trabajo, que puede resolver, en forma autónoma y flexible, los problemas que se le presenten en el ejercicio de sus funciones, y está capacitado para colaborar en su entorno profesional y en la organización de su trabajo.

En este recorrido es interesante ver cómo la significación del término puede denotar flexibilidad y extensión, en tanto un contexto más amplio que el laboral. Sin embargo, la mayoría de veces se extrapola a ámbitos que terminan enmarcándose con la práctica profesional y laboral; es el caso de Irigoin y Vargas (2002), para quiénes la competencia es la 
combinación integrada de conocimientos, habilidades y actitudes que se ponen en acción para un desempeño adecuado en un contexto dado. Aún más, se explicita sobre un saber actuar movilizando todos los recursos que posee el individuo. Mientras que para Navío (2002), una competencia es el conjunto de elementos combinados: conocimientos, habilidades, actitudes, valores y normas, entre otros, que se integran sobre la base de una serie de atributos personales, tales como capacidades, motivos, rasgos de personalidad y aptitudes, tomando como referencia las experiencias personales y profesionales y manifestándose mediante determinados comportamientos o conductas en el contexto de trabajo.

Ahora bien, en el ánimo de un estudio más detallado en análisis del concepto de competencias en la educación superior, los diferentes enfoques teóricos pueden ser catalogados en dos corrientes de pensamiento de la siguiente manera: la corriente crítica en la cual están los teóricos más radicales, y la otra, la corriente empirista, vertiente relacionada con la óptica del mercado, los enfoques empresariales y el modelo conductual.

En la primera corriente se sitúan autores como Gimeno Sacristán (2008), quien plantea que el hecho de formular la educación en torno a competencias claves responde a determinados intereses de determinadas organizaciones, fundaciones y organismos internacionales, entre otros. Basándose en los planteamientos del proyecto DeSeCo advierte, además, que este nuevo enfoque invierte el planteamiento clásico del saber hacer, de forma que lo evaluable y lo evaluado pasa a convertirse en la finalidad a la que habrá que prestar atención para planificar y desarrollar el currículo.

Tanto en las competencias que plantea la Unión Europea como las presentadas por el proyecto internacional DeSeCo se reconoce la dificultad de estructurar el currículo en torno a ellas, dada su proximidad al contenido del currículo. No obstante, advierte sobre la necesidad de saber cuáles deberían ser esas competencias; se debe llegar a un acuerdo elemental y fundamental en torno al propio significado sobre qué son las competencias.
Para Pérez Gómez (2008) el término competencia tiene una fuerte influencia conductista, se asocia con las habilidades y se relaciona con el concepto de conocimiento práctico, con unas características bien distintas que exigen reinventar la escuela en su forma de enseñar, aprender y evaluar. Martínez Rodríguez (2008) considera que las competencias son comportamientos y capacidades definidas por otros para actuar de manera determinada. Por tanto, se reduce la autenticidad e indeterminación de la acción humana y se piensa en el desarrollo de la mente como un mero resultado que lleva a una interpretación limitada de la educación.

En tal sentido, en el discurso de las competencias a menudo se ha ido dejando a un lado la dimensión sociológica que el concepto engloba, dando prioridad a aspectos de orden cognitivo y psicológico. Otras de las críticas se relacionan con las connotaciones éticas y políticas que conlleva el discurso, así como las referidas al carácter individualista y disciplinar de las competencias.

Para Torres Santomé (2008) existe diversidad de significados sobre el término competencia ${ }^{10}$. Este hecho puede resultar peligroso e ineficaz a la hora de una mayor concreción; la tecnocracia del discurso que hace uso de múltiples conceptos aparentemente consensuados, utilizados por organismos supranacionales. No obstante, con el concepto de competencias se pretenden cambios metodológicos, pero no se plantea el modelo de sociedad que queremos construir, ni se consideran las condiciones en las que el profesorado desempeña su labor porque existe un idealismo en el discurso que presupone un mundo social abstracto en el que no rigen posiciones de poder ni control de la cultura. De otro lado, se olvida el conocimiento y los contenidos culturales. Por ello, desde sus orígenes se trata de una política educativa claramente orientada hacia los sectores productivos.

Resulta interesante la perspectiva de Angulo Rasco (2010), quien manifiesta que el movimiento de las competencias en educación superior ha sido

10 Concepción derivada del estudio del proceso educativo español. 
incorporado a nuestro vocabulario y a formas de acción sin un cuestionamiento previo. El autor se centra en la concepción mercantilista del concepto y en sus implicaciones en el proceso de diseño de perfiles profesionales. En este proceso destaca la concepción mecánica y uniforme del profesional, el bloqueo de las dimensiones sociales, culturales, valorativas y políticas de la formación y la enseñanza, o en el significado meramente instrumental de la educación. De esta manera, el significado mercantilista se justifica en la necesidad de la adaptación de los futuros universitarios al mundo laboral y las demandas actuales del mercado.

Otro autor de gran importancia del enfoque crítico es Díaz Barriga (2006), quien manifiesta que el término competencia tiene un sentido utilitario en el mundo del trabajo. En él se articula, por una parte, algo que es consecuencia de un desarroIlo natural (visión Chomskiana), con un sentido claramente utilitario (contradice la necesidad de adquirir o mostrar conocimientos), para dar cuenta de la necesidad de resolver problemas. Lo utilitario sobre lo erudito, lo utilitario como elemento específico de la llamada sociedad del conocimiento.

La segunda corriente, la empirista, se encuentra relacionada con la lógica del mundo del mercado, la lógica mercantil: allí se puede hacer referencia a los organismos supranacionales como el Proyecto Tunning (2000-2001), en donde las competencias presentan una combinación de atributos que describen el nivel o grado de suficiencia con que una persona es capaz de desempeñarlos. En la misma dirección, Arguelles y Gonczi consideran que "es necesario contar con normas de competencia consensuadas y adecuadas a las necesidades de la industria, que sirvan de base a la educación y a la capacitación vocacional" (2000, p. 56). Por tanto, la educación basada en normas de competencia no solo desempeñará un papel importante en el desarrollo de la competitividad económica, pues competencia significa organización en criterios de atributos, desempeños y tareas claves.

De otro lado, Perrenoud (2005) define la competencia como la aptitud para enfrentar eficazmente una familia de situaciones análogas, movilizando a conciencia y de manera rápida, pertinente y creativa, múltiples recursos cognitivos: saberes, capacidades, micro-competencias, informaciones, valores, actitudes, esquemas de percepción, de evaluación y de razonamiento, categoría compartida con el Proyecto DeSeCo (2002) que como ya se ha dicho, concibe las competencias como la capacidad de responder a demandas complejas. De esta manera, una competencia se define como la habilidad para hacer frente con éxito a las demandas complejas en un contexto particular a través de prerrequisitos psicosociales como lo plantean Rychen y Salganik (2003). El conjunto de estos enfoques resulta ampliamente relacionado con un sentido de los efectos de la educación, la capacitación y el aprendizaje. Situándose en una postura intermedia de las competencias que reconocen sus ventajas y desventajas como enfoque, sin ser empiristas.

Otros conceptos de competencia derivados de este enfoque son el propuesto por la OIT (2004) desde el cual se reconoce la competencia como la capacidad de llevar a cabo o ejecutar conocimientos diversos de forma adecuada. Dicha concepción supone una combinación de habilidades prácticas y conocimientos, motivación, valores éticos, actitudes y emociones, sumados a otros componentes sociales y de compromiso que se movilizan conjuntamente para lograr una acción eficaz y efectiva para llevar a cabo exitosamente una actividad laboral plenamente identificada y planificada.

De esta manera, en la lógica del empleo y la perspectiva de cambio, que recurre al sistema de aprendizaje diferente, se puede identificar las líneas de pensamiento que se exponen a continuación.

De un lado, Montmollin (1984), citado por Ceinos (2004), refiere la competencia como aquellos rasgos personales, conductas o acciones concretas que son examinados desde el "conjunto estabilizado de saberes y saber-hacer, de conducta, tipo de razonamiento, que se pueden poner en práctica sin nuevo aprendizaje" (p. 96). Por otro lado, para Reyes (2001) una competencia es la aptitud de una persona, para desempeñar una misma función 
productiva en diferentes contextos de trabajo con base en los resultados esperados. En tal sentido, la competencia es equivalente a saber actuar en forma autónoma e incluye: saber escoger, tomar iniciativas, arbitrar, correr riesgos, reaccionar ante lo imprevisto, contrastar, tomar responsabilidades y saber innovar; de tal forma, la competencia como construcción, parece ser el resultado de una combinación pertinente de varios recursos (Le Boterf, 2000).

En otra concepción, Mateo (2007) define la competencia como la capacidad de usar funcionalmente los conocimientos y habilidades en contextos diferentes, situación que implica comprensión, reflexión y discernimiento, donde es necesario tener en cuenta, de forma simultánea e interactiva, la dimensión social de las acciones a realizar. En la misma línea, García Sanz y Morillas Pedreño (2011), se refieren a la capacidad para seleccionar y movilizar conocimientos, habilidades y actitudes para responder con éxito a una determinada situación profesional; complementa su definición citando las palabras de Collins (2007), quien concibe la competencia como la integración de conocimientos, habilidades y actitudes de forma que nos capacite para actuar de manera efectiva y eficiente.

Por ello, y a modo de resumen, indistintamente, de la postura, enfoque y contexto, "las competencias, sean del orden que fueren, seguirán siendo comportamientos y capacidades para actuar de manera deseadas y definidas por otros" (Barnett, 2001, p. 122). Por tanto, la formación basada en competencias obliga inevitablemente a replantear las nociones y tradicionales fijadas por el currículo, por ello, vale la pena reflexionar respecto a su concepto y su relación con la educación superior.

\section{Contexto latinoamericano y las competen- cias en educación superior}

El pensar en las posibilidades reales de la formación por competencias en educación superior y el trabajo profesional para los jóvenes universitarios, a propósito del sentido de formación de las nuevas estructuras universitarias, amerita una reflexión importante, respecto a temas relacionados con las competencias, la formación por competencias en la universidad y situaciones-problema asociados.

En América Latina, como ocurrió hace más de una década en Europa, se asiste "a una lenta transformación de las estructuras universitarias" (Mira Solves et al., 2012, p. 447). En diferentes latitudes del continente se trabaja por construir una teoría basada en una lógica de competencias bajo una perspectiva curricular, argumentando que repercutirá en la mejora del empleo, la competitividad, la innovación, el desarrollo cultural y en el aumento del nivel general de la educación superior de la población.

Ahora bien, desde un marco de acción mundial, se hace inevitable reconocer que:

[...] los sistemas educativos de la región nunca estuvieron estáticos. Así, durante el siglo XX, específicamente, en la década de los 60', existieron y persisten, en América Latina, una serie de políticas y estrategias efectivas en la determinación de un cambio en las relaciones entre el centro de conducción de los sistemas educativos y en cada una de las instituciones que ofrecen servicios educativos. (Braslavsky y Cosse, 2006)

Luego de la aparición del Proyecto Principal sobre Educación (Promedlac), firmado por los países latinoamericanos en 1981, se concentró en dar cumplimiento a grandes objetivos como el de alcanzar la educación básica para todos, alfabetizar a la población joven y adulta, completar las reformas necesarias para lograr la calidad y aplicar con efectividad acciones para el fortalecimiento de la educación.

En este sentido, durante la década de 1990 en el mundo se realizaron acciones políticas para modernizar y transformar los sistemas educativos de los diferentes países en el mundo; gestiones reflejadas en iniciativas tales como la conferencia mundial de Jomtien, Conferencia Mundial de Educación para todos y la reunión en Ammán, Jordania, a fin de evaluar los progresos y así dar cuenta de 
los logros alcanzados. De manera conjunta, en el comunicado final del Foro aprobado con el título Reafirmación de Ammán, se declaraba que en los seis años transcurridos desde Jomtien "han habido avances significativos en el ámbito de la educación básica, aunque no en todos los países ni tantos como se habían esperado" (Unesco, 2000, p. 7).

Para la misma época se encuentra un listado de eventos relacionados con la educación, entre los que se destaca: La Cumbre Mundial en favor de la Infancia (1990), La Conferencia de las Naciones Unidas sobre Medio Ambiente y Desarrollo (1992), La Conferencia Internacional sobre la Población y el Desarrollo (1994), La Conferencia Mundial sobre Derechos Humanos (1993), La Conferencia Mundial sobre Necesidades Educativas Especiales: Acceso y Calidad (1994), La Cumbre Mundial sobre el Desarrollo Social (1995), La Cuarta Conferencia Mundial sobre la Mujer (1995), La Conferencia Internacional sobre Educación de Adultos (1997) y la Conferencia Internacional sobre el Trabajo Infantil (1997), entre otros acontecimientos:

[...] patrocinados por cinco organismos: el Programa de las Naciones Unidas para el Desarrollo (PNUD), la Organización de las Naciones Unidas para la Educación, la Ciencia y la Cultura (Unesco), el Fondo de Población de las Naciones Unidas (FNUAP), el Fondo de las Naciones Unidas para la Infancia (Unicef) y el Banco Mundial. (Unesco, 2000, p. 8)

En la misma dirección se encuentran Las Cumbres Iberoamericanas de Jefes de Estado y de Gobierno, las Asambleas de Jefes de Estado y de Gobierno de la Organización para la Unidad Africana y otras; también se constituyeron foros de concienciación de los gobiernos acerca del papel fundamental que juega la educación, tal como lo señaló Fernández Santamaría (2000):

[...] los discursos parecieron tener "la intención de refundar las formas a través de las cuales las sociedades satisfacen sus necesidades educativas, indicadas desde las nuevas leyes de educación general o la adopción de amplios planes nacionales de reforma, que incluyen principios y propuestas organizacionales, administrativas, curriculares y pedagógico-didácticas [...] (como se cita en Braslavsky y Cosse, 2006)

El año 2000 comenzó con las iniciativas de Dakar en Senegal, para aprobar el Marco de Acción y el compromiso con los seis objetivos mundiales, entre los que se encuentra la ampliación de los servicios de educación básica y de capacitación a otras competencias esenciales para jóvenes y adultos; la Cumbre del Milenio y su Declaración del Milenio de las Naciones Unidas para unificar voluntades y compromisos para la cooperación internacional con los países menos desarrollados, luchar por la desigualdad, mejorar el acceso a la educación, promover la igualdad de género y combatir la pobreza extrema, entre otros objetivos.

A nivel de Latinoamérica estuvo el Marco de Acción Regional, Educación para Todos en las Américas, celebrado en febrero del 2000, con el fin de renovar los compromisos de Educación para Todos, para los próximos 15 años; la Reunión del Proyecto Principal de Educación; así mismo en América Latina y el Caribe-Promedalc se celebró en Cochabamba, Bolivia; la Declaración de la Habana, reunión Intergubernamental del Proyecto Regional de Educación para América Latina y el Caribe, PRELAC en noviembre 2002. De igual manera, en las últimas Conferencias Iberoamericanas de Educación, los gobiernos han dado cuenta de los esfuerzos significativos a favor de la educación, es el caso de la XXI Conferencia Iberoamericana de Educación: Transformación del Estado y Desarrollo, cuyo manifiesto da cuenta de la importancia de la educación para formar ciudadanos libres, tolerantes, cultos y solidarios, para favorecer el desarrollo económico y social y para garantizar el fortalecimiento de nuestras instituciones democráticas. 
En la XXII Conferencia Iberoamericana de Educación, el lema fue La Formación Técnico-Profesional y su impacto en el tejido empresarial, convocada en el marco de la XXII Cumbre Iberoamericana de Jefes de Estado y de Gobierno, en el cual se acordaron entre otras prioridades: renovar el compromiso adquirido al aprobar el programa Metas Educativas 2021, ratificado por los Jefes de Estado y de Gobierno en la Cumbre de Mar del Plata (Argentina) en el año 2010, de que en el año 2015, entre el $40 \%$ y el $70 \%$ de los alumnos culminen la educación secundaria superior y entre el $60 \%$ y el 90 \% lo hagan en 2021.

En la esfera norte, no obstante que la ley resultó ineficiente a pesar de su sentido de descentralización, se conoció:

[...] el Informe del Progreso Educativo en Estados Unidos, presentado en la década de los 80, el ex-presidente George W. Bush, propuso el Plan América 2000, a fin de reformar el sistema educativo y orientarlo hacia la competitividad y las competencias de los educandos. Dichas reformas se concretaron con la firma de la Ley "Que Ningún Niño Se Quede Atrás" (No Child Left Behind Act) en enero de 2000. (Garibay, 2011)

En Europa, en la última década se ha logrado un consenso regional sobre reformas y políticas educativas a través de la publicación de documentos que en conjunto conforman el marco propositivo del Espacio Europeo de Educación Superior (EEES).

En 1997 se firmó el Convenio de Lisboa; en 1998, los Ministros de Educación de Francia, Reino Unido e Italia aprobaron la Declaración de la Sorbona, para la Armonización de la Arquitectura de la Educación Superior Europea; en el 2001 el Comunicado de Praga (para la promoción del aprendizaje a lo largo de la vida, implicación de los estudiantes en la educación superior, incremento atractivo de la educación superior europea); en 2003, el comunicado de Berlín, y en 2005, el Comunicado de Bergen. (Angulo Rasco, 2010)
El Proyecto Tuning Education Structures in Europe, creado para continuar cumpliendo con el encuentro conjunto en la universidad europea y lograr la exploración de consensos, el respeto a las diversidades, la transparencia y la confianza mutua para llegar conjuntamente a puntos de referencia comunes. Puntos de referencia basados en resultados del aprendizaje, competencias, habilidades y destrezas que no tienen carácter normativo sino referencial (Universidades de Deusto y Groningen, 2003); ofreciendo el modelo de diseño curricular esencial, estandarizado, válido y eficaz que posibilitará comparar titulaciones así como el establecer criterios de acreditación y evaluación estándar (Angulo Rasco, 2010).

En términos generales, puede decirse que la tradición académica ha podido, en el tiempo, realizar internamente diferentes interpretaciones de la realidad y traducirlas, de algún modo, en sus procesos de planificación, enseñanza, investigación, evaluación, entre otras. Así, las reformas educativas más actuales son transferidas por lo general desde afuera e impuestas desde organismos multilaterales y referenciadas con expresiones tales como "la lógica de competencias, la perspectiva socio-constructivista, la atención centrada en los alumnos, y la importancia creciente de las situaciones de formación" (Jonnaert et al., 2008, p. 9), propuestas que en muchas oportunidades se ven relacionadas con "las lógicas del mercado, la empresa y la burocracia, sin considerar sus propias condiciones y sus contribuciones al mundo de la cultura, del trabajo y a la urgente problemática de las formas y de los métodos de la producción" (Mockus, 2012b, p. 51).

Es así como el papel de la Universidad en América Latina se ha visto influenciado por las tendencias de transformación y reforma educativa presentadas con antelación, especialmente en el ámbito del currículo y, para el caso particular, el currículo por competencias. Sin embargo, algunos aspectos como la gestión están más considerados a nivel político, otros permanecen significativamente ausentes, caso de la postura pedagógica, la 
formación y capacitación docente, o, simplemente, son marcos del tratamiento de otro conjunto de intenciones.

\section{Brevemente el caso colombiano}

Con base en el apartado anterior y desde una perspectiva crítica es importante puntualizar que:

[...] las competencias constituyen un concepto del discurso de la calidad de la educación superior colombiana que carece en gran medida de reflexión pedagógica, conceptual, epistemológica y filosófica, motivo por el cual este concepto puede acomodarse a diferentes intenciones comunicativas o propósitos gubernamentales. Esto se observa en la ausencia de investigaciones y publicaciones reflexivas tanto por parte del Estado como por parte de las universidades, pues es tan común que no suscita discusión. (Tobón, 2008, p. 4)

Sobre este sello, comprender la especificidad del marco regional colombiano requiere tener en cuenta las políticas educativas relacionadas con el tema y la incidencia de algunos organismos internacionales que trabajan para su mejoramiento. La política de Estado, expresada en la Política Nacional para la Educación, consiste en seguir ofreciendo más oportunidades de cursar estudios de educación superior y aumentar las tasas de graduación (OCDE, 2012). Sin embargo, las recomendaciones de evaluadores y expertos hacen notar que el mecanismo hasta ahora dispuesto no es suficiente para lograrlo, demostrando una vez más que la decisión educativa responde a presiones irresistibles de organismos internacionales antes que a la satisfacción de necesidades internas.

En Colombia la Ley 30 de diciembre 28 de 1992 organizó el servicio público de la educación superior. No obstante, en la actualidad la Ley 30 se encuentra en proceso de reformulación y debate. Durante los últimos 10 años, decretos tales como el 749 y 808 de 2002, el 2566 de 2003 y 1188 de 2008 han establecido condiciones respecto a la calidad y pertinencia de programas en educación superior. Por ejemplo, el Decreto 2566 de 2003 estableció las condiciones mínimas y demás requisitos para el ofrecimiento de programas académicos de educación superior; pero, dicha disposición fue modificada por el Decreto 2170 de 2005, en el cual el artículo $4^{\circ}$, sobre aspectos curriculares, se plantea el "desarrollo de competencias y habilidades de cada campo y área de formación y el desarrollo de competencias contempladas en los perfiles de formación de cada programa" (Ministerio de Educación Nacional, 2005).

El Plan Estratégico del Sector Educativo 20112014 del Ministerio de Educación Nacional contempla entre sus objetivos y estrategias, en materia de educación superior, el fortalecimiento del desarrollo de competencias genéricas y específicas; indicando así la reestructuración de los currículos y actualización de los planes de estudio universitarios (MEN, 2011). Así mismo, otras disposiciones del sistema educativo colombiano dejan ver su asentimiento hacia el modelo competencial, tal es el caso de: el sistema de examen aplicado por el Instituto Colombiano para la Evaluación de la Educación (Icfes), que mide las competencias de los estudiantes al entrar y salir de la educación superior; la participación en las pruebas internacionales como Pisa (Program for International Student Assessment, por sus siglas en inglés) o TIMSS (Trends in International Mathematics and Science Study), de la Asociación Internacional para la Evaluación del Desempeño Educativo, cuyos resultados son considerados desde el 2012 como primordiales para la creación de políticas públicas según la OCDE.

El informe sobre la educación superior en Colombia 2012, realizado por el programa de trabajo de la Dirección de Educación de la OCDE, en colaboración con el Banco Mundial, ofrece un estudio profundo del sistema de educación y propone una serie de recomendaciones sobre el 
acceso y la equidad, la pertinencia, la gobernabilidad, la gestión, la investigación, la medición del aprendizaje, la integración, el financiamiento, los mecanismos de aseguramiento de la calidad y la promoción de la integración internacional para la educación superior colombiana (OCDE, 2012). En otro de sus apartados, el informe hace mención al tema en cuestión expresando que:

[...] muchas instituciones no tienen más que escasos vínculos y colaboraciones con los empleadores sobre el desarrollo del currículo, las competencias y los resultados deseados, lo cual limita la pertinencia de sus programas con las necesidades de la economía colombiana y puede hacer que sus egresados tengan menos posibilidades de empleo. (OCDE, 2012, p. 59)

En este sentido, el equipo formulador del informe en el tema de calidad y pertinencia de los programas, encuentra acertado el empeño del Ministerio de Educación Nacional por desarrollar un enfoque basado en las competencias, tanto en los sistemas de educación como de formación para el trabajo, teniendo en cuenta el nivel de competitividad de Colombia. Sin embargo, tras las reuniones con instituciones de educación superior (IES) y el sector empresarial se concluyó que el aporte de los empresarios al diseño curricular y a la identificación de las competencias debe ser fortalecido considerablemente en las universidades públicas (OCDE, 2012).

En este contexto, la formación por competencias se ha desarrollado y articulado a partir de la organización de la educación acorde con las reglas del mercado y las competencias se convierten en indicadores de calidad que son controladas por pruebas estandarizadas. De esta forma, la lógica impuesta por el poder político y económico del país ha posibilitado la formación por competencias a través de una serie de reformas al sistema educativo con unos propósitos marcados frente a tres aspectos fundamentales: a. Hacer un país más competitivo y que responda a los retos de mercado en un mundo en un marco de globalización económica.

I. Disminuir la brecha efectiva entre la formación profesional y el mundo laboral.

$\mathrm{m}$. Asumir el reto de formar estudiantes que puedan incorporarse y adaptarse a las variadas ocupaciones y profesiones que pueden asumir en su vida laboral.

Sobre estos aspectos es importante analizar el caso de las competencias en los currículos universitarios que se desarrolla en el siguiente apartado.

\section{Competencias y currículo universitario}

A la hora de formalizar el currículo para la educación general se tropieza con un problema serio: la enorme dificultad de identificar y seleccionar las funciones y papeles vitales generales (De la Orden Hoz, 2011), no hay que olvidar que los cambios importantes que tienen lugar en este se interpretan como "la continuidad con un pasado y con las relaciones entre sociedad y educación superior" (como se cita en Barnett, 2001, p. 101).

En los últimos tiempos, distintas universidades latinoamericanas han cambiado sus modelos educativos tradicionales por uno centrado en el aprendizaje, un modelo curricular flexible o semiflexible, un modelo basado en competencias profesionales.

El currículo se puede considerar como un proceso educativo integral que permite rediseñarse sistemáticamente en la medida en que se producen cambios sociales. Por ello, el currículo "tiene que ser una clasificación de conocimientos pero también un proyecto epistemológico" (Barnett, 2001, p. 75). De tal forma que hay quienes consideran que los requisitos que debe cumplir un buen diseño curricular en su elaboración, ejecución, control y evaluación son: consideración de los ejes transversales y programas directores; integración de los componentes académico, laboral e investigativo; 
participación de profesores y alumnos en su evaluación y elaboración; correspondencia con las necesidades educativas de la enseñanza y nivel; contribución a la formación de valores políticos en los estudiantes y aseguramiento de la base material de estudio (Guerra, 2001).

En este sentido se tiene que "los responsables de los sistemas educativos se vienen interesando por los contenidos del aprendizaje escolar, currículo y programas de estudio, buscando nuevos referentes para la reformulación de los mismos" (Jonnaert, 2008, p. 4); así, para organizar los currículos existen las estructuras por asignatura, por problemas y por competencias. Sin embargo, y a pesar de sus intencionalidades, en las prácticas educativas universitarias la modelo tradicional continua vigente; en contrapartida, las propuestas curriculares recientes incorporan ciertos principios innovadores.

Una postura que respalda el asentamiento de lo que es el currículo por competencias es la de Rial Sánchez (2011) quien, partiendo de la obra de Franklin Bobbit, realiza una doble definición del currículo. Por un lado, lo entiende como todas aquellas experiencias encaminadas a desarrollar las habilidades del individuo (sean estas dirigidas o no); y por otro, como aquellas experiencias aplicadas en las escuelas y dirigidas de forma consciente a alcanzar ese desarrollo en los alumnos. Este planteamiento defiende la postura que el currículo debe preparar a los alumnos para el ejercicio de la vida adulta y la toma de decisiones que esta conlleva, la que está caracterizada por el trabajo y la formación que ello implica.

No obstante, Lawrence Stenhouse (1975), citado por Kemmis (1993), afirma que el currículo es un intento por comunicar los principios esenciales de una propuesta educativa de tal forma que quede abierta al escrutinio crítico y pueda ser traducida efectivamente a la práctica. Mientras que algunas otras definiciones de currículo lo hacen ver como "un conjunto de principios sobre cómo deben seleccionarse y transmitirse el conocimiento y las destrezas" (Lundgren, 1991), o "un proyecto educacional que define: los fines, las metas y los objetivos de una acción educacional así como las formas, los medios y los instrumentos para evaluar en qué medida la acción ha producido efecto" (D'Hainaut, 1980). La Unesco, en el año 1966, lo definió como "la organización de un conjunto de experiencias de aprendizaje y los diversos factores que las condicionan y determinan, en función de los objetivos básicos generales o finales de la educación" (Vila Morales, 2011).

Ahora bien, el currículo desde el ámbito social refleja una práctica cotidiana institucional organizada que es, a la vez, compleja, dinámica, colaborativa y que, desde el ámbito político, al vincularse la escuela con la sociedad, genera proyectos en un proceso de toma de decisiones reflexiva y crítica (Ferreyra et al., 2008). Situación que puede entenderse como contradictoria cuando se asume de forma instrumental el discurso de las competencias; es decir, cuando son admitidas como un conjunto de habilidades y conocimientos aplicados a una función ocupacional, a partir de requerimientos establecidos por el empleo (Pavié, 2011).

Por ello, Gimeno Sacristán señala que:

[...] los debates esenciales en torno a los currículos en la actualidad, como no podía ser de otro modo, están muy estrechamente relacionados con los cambios culturales, políticos, sociales y económicos que están afectando a las sociedades desarrolladas y que tienen como primera consecuencia la revisión del papel asignado a la escolarización y a las relaciones entre esta y los diferentes aspectos que en ella se entrecruzan: profesores, organizaciones, relaciones con la comunidad, etc. (2001, p. 14)

De esta manera, el estado actual de la economía, el factor político en el mundo y las complejidades del desarrollo sociocultural determinan los trazados educativos de la educación en el mundo contemporáneo. No obstante, más allá del discurso de las competencias los currículos universitarios deben asumir una crítica frente a las complejidades del contexto, esto es, de las 
determinaciones sociales, económicas y productivas que traza la gran empresa, así como mantener el rumbo de la formación integral que supere el simple entrenamiento para desarrollar habilidades y destrezas que se realizan en el mundo laboral - las competencias centradas exclusivamente al desempeño de una profesión ${ }^{11}$ - .

Sobre esta lógica, a pesar del desarrollo global y de la puesta en escena de la política internacional sobre el tema de las competencias en la educación superior cabe un papel esencial soportado en la autonomía universitaria. Pues las universidades entran en debate cuando trazan sus proyecciones formativas a través de la configuración de su proyecto educativo institucional, en el que también definen sus modelos curriculares ${ }^{12}$ que pueden ser de diversa índole. En esta dirección, existe una tendencia que traza tres modelos bastante definidos para los currículos de la educación superior a saber: el primer modelo (con mayor expansión en su proceso de adaptación) se caracteriza porque integra las competencias genéricas o transversales con las específicas de las áreas disciplinares (de las cuales se profundiza en el mundo laboral actual), entre ellos están los establecidos en organizaciones y países como: DeSeCo/OCDE, Tuning, Alemania, Bélgica, Holanda, Dinamarca, Irlanda, Grecia, Luxemburgo, Suecia, Austria, Portugal, Reino Unido y la Comunidad Autónoma de España: País Vasco. De otro lado, existen los modelos curriculares en los que las competencias básicas no se diferencian de las áreas disciplinares como son los modelos curriculares de Finlandia e Italia. Por último, tenemos los denominados modelos curriculares mixtos, en los que se coarticulan competencias transversales y las competencias de

11 Para Cano (2005) existen dos tipos de competencias: las básicas o transversales que incorporan las competencias intelectuales e interpersonales, y las específicas que se derivan de un contexto o trabajo específico (profesional o académico).

12 En este orden, las universidades vislumbran los objetivos de formación, los perfiles ocupacionales de sus egresados, el modelo curricular, las perspectivas pedagógicas y didácticas, los contenidos y su nivel de profundidad, entre otros aspectos que robustecen el conjunto de capacidades, habilidades que los estudiantes deben adquirir, desarrollar y demostrar en el ejercicio de su profesión las áreas disciplinares. Este es el tipo de modelo adoptado por la Comisión Europea, Dinamarca, Austria, Portugal, España (LOE), Luxemburgo, Francia y Generalitat de Cataluña (España).

La presencia de estos modelos ha permitido identificar los modos como las universidades han transformado sus modelos curriculares de forma paulatina, pues ha girado del dominio sujetado a la práctica profesional hacia planes de estudio que cuentan con escenarios educativos que benefician las tareas prácticas, de la profesionalización y la diversificación de los saberes en las distintas disciplinas: por ello, existe una mayor preocupación en los futuros desempeños que en los contenidos que determina la estructuración de los planes de estudio.

Sobre esta lógica se concibe que la formación profesional universitaria no puede perder el norte en sus procesos de formación de profesionales. El currículo en educación superior debe ahondar en sus componentes antropológico, filosófico y epistemológico desde una perspectiva humanista, social y cultural. Aunque el currículo en la educación superior esté basado en competencias, no se puede dejar de lado la reflexión y discusión sobre los diferentes aspectos teóricos, epistemológicos, metodológicos, sociales y culturales relacionados con los problemas globales y específicos que se plantean como punto de partida para la reconstrucción y apropiación de los saberes en el proceso de formación de profesionales. Así, la estructura curricular tendrá como finalidad la comprensión de la cultura y de la práctica laboral, lo que posibilita un desarrollo integral de los nuevos profesionales capaces de leer los contextos y desafíos que enfrenta el mundo del trabajo. De otro lado, los procesos de formación, pensados de esta manera, deben consolidar la autorreflexión como medio adecuado para la constitución de sujetos críticos, participativos y comprometidos con las realidades sociales, económicas y políticas a partir de la construcción colectiva del conocimiento. Se asume entonces que el diseño curricular basado en competencias debe superan las prácticas laborales 
tradicionales y el cumplimiento de requisitos trazados por las normas laborales que determina en mundo empresarial.

\section{Reflexiones finales}

El cambio de mirada de las instituciones de educación superior como reto educativo les exige repensarse y reinventarse con "una posición respecto del papel que desempeñarán en el futuro" (Altbach, 2009, p. 36). Sin embargo parte de la reflexión consiste en cuestionarse, entre otras cosas, si la lenta transformación de las estructuras universitarias ha modificado en la misma lógica el sentido y función misional institucional, y en esa vía, si al asumir esos nuevos procesos de formación, específicamente bajo una perspectiva curricular de formación por competencias, realmente repercutirá en la mejora del empleo, la competitividad, la innovación, el desarrollo cultural y en aumento del nivel general de la educación superior.

El modelo por competencias, como se enunció en otros apartados, no es una forma o fórmula nueva; su materialización requiere de la construcción de una propuesta curricular coherente a su intención, en tanto su naturaleza, característica poblacional, contexto cultural, interpretación política y, por qué no, poder burocrático. Aunque se sigan haciendo ver como eje innovador para la formación universitaria contemporánea, en la mayoría de docentes se percibe como un planteamiento con tradición y contradicción que adolece de carácter científico y pedagógico porque desde hace varios años se viene introduciendo en algunas reformas y experiencias universitarias.

Desde un carácter pedagógico, se hace necesario repensar en el mismo campo situaciones problemas, tales como: los procesos de reconstrucción de la docencia, la transformación de la institución educativa a nivel curricular, administrativa y física; las adaptaciones e implementaciones de los sistemas didácticos y evaluativos que la perspectiva exige.
Las competencias como un lenguaje o discurso innovador, introduce un vocabulario con limitaciones y concepto diversos, confusos para manejarlo en el campo científico y no necesariamente se puede llevar a la práctica o vida real; si hay una nueva terminología en el universo conceptual de la formación, debe haber nuevas luces y formas epistemológicas que lo sustenten. En ese plano, las competencias no lo son; en cambio, como se ha mencionado, se originan en fenómenos mercantiles para demostrar la eficacia de una inversión económica, débil en elementos para convertirse en enfoque de formación:

[...] la educación por competencias, el currículo flexible, las tutorías, el aprendizaje basado en problemas y casos, la formación en la práctica, el currículo centrado en el aprendizaje del alumno y otros más, se aglutinaron bajo la etiqueta de modelos innovadores. (Díaz-Barriga Arceo, 2010, p. 39)

De otro lado, la construcción participativa del currículo desde las perspectiva de las competencias requiere de la colaboración de todos los actores involucrados en el proceso de enseñanza-aprendizaje, pero la situación muestra que el nivel de participación de los actores en los procesos de reforma curricular es muy baja, a pesar de que se habla de proyectos participativos: "la elección u orquestación de las innovaciones que se introducen en el currículo procede de los expertos en contenido o de los diseñadores curriculares, por lo que en muchos casos se sigue trabajando en una lógica de implantación unidireccional" (Díaz-Barriga Arceo, 2010, p. 40). Esto mientras se deja a los docentes el cumplimiento de la innovación, obligándolos de manera implícita y explícita a un cambio en su práctica, ignorando cuestiones relacionadas con el:

[...]cómo aprenden los profesores, qué los impele a cambiar o no sus prácticas educativas, qué procesos ocurren cuando se enfrenta la tarea de innovar o qué condiciones se requieren para que 
un cambio real ocurra y se consolide. (Díaz-Barriga Arceo, 2010, p. 38)

Ahora, desde el punto de vista curricular, para que las personas sean competentes, ¿se debe formar en un currículo por competencias forzadamente? ¿Qué pasa con las situaciones formativas que no están dentro de la lógica de las competencias, es decir, aquellos terrenos distintos a las formulaciones o expresiones competentes que otros consideran como tal?

Las competencias se pueden elevar a los problemas precisos, con aprendizajes concretos y delimitados, pero cuando en la formación se adentra en el para qué del conocimiento y en casos que van más allá de lo meramente técnico, es decir, lo relacionado con lo que no es posible verificar y cuantificar pero que existe (y no se hace referencia a los valores), como condiciones de expresión humana, y es susceptible de fortalecerse entre humanos; aquello que se vincula con el qué del conocimiento, las relaciones entre enseñanza aprendizaje, los maestros, traducido en las intencionalidades pedagógicas y no solo en el sentido de lo aplicabilidad: actitud, capacidad y eficiencia.

Si la formación por competencias es una innovación, tal vez requiera un cambio de paradigma importante. Sin embargo, las evidencias desde el punto de vista de la investigación son pocas, pues son contados los resultados al respecto:

[...] la percepción compartida entre profesores y estudiantes que la evaluación de competencias comporta, como aspectos positivos, la mejora del aprendizaje, estimando que más allá de las formas concretas, se centra en el aprendizaje del estudiante y en la reflexión sobre los procesos innovadores que el docente lleva a cabo para ello. (Tierno García, 2011).

La formación por competencias no se puede centrar en términos del aprendizaje que se pueda confirmar. Las nuevas nociones relacionadas con el currículo por competencias expresan un constructo mental que, desde el punto de vista metodológico, debe ser predecible, medible, constatable, además de deseadas y definidas por otros.

\section{Referencias}

Altbach, P. (2009). Educación superior comparada. El conocimiento, la universidad y el desarrollo. Buenos Aires: Universidad de Palermo. Colección de Educación Superior.

Angulo Rasco, J. (2009). Educar por competencias, ¿qué hay de nuevo? Madrid: Morata.

Angulo Rasco, J. (2010). La voluntad de distracción: las competencias en la universidad. Madrid: Morata.

Arguelles, A. y Gonczi, A. (2000). Competency based education and training: a world. Mexico: Conalep/ Noriega.

Barnett, R. (2001). Los límites de la competencia: el conocimiento, la educación superior. Barcelona: Gedisa Editores.

Barrick, M. y Mount, M. (1991). The big five personality dimensions and job performance: A meta-analysis. Personnel Psychology, 44(1), 1-26. https://doi.org/10.1111/j.1744-6570.1991.tb00688.x

Blas Aritio, F. (2007). La formación profesional basada en la competencia. Avances en supervisión educativa. Revista de la Asociación de Inspectores de Educación de España, 7, 19-20.

Boon, J. y Van der Klink, M. (2002). Competencies: The triumph of a fuzzy concept. Academy of $\mathrm{Hu}$ man Resource Development Annual Conference, 327-334.

Braslavsky, C. y Cosse, G. (2006). Las actuales reformas educativas en América Latina: Cuatro actores, tres lógicas y ocho tensiones. Revista Electrónica Iberoamericana sobre Calidad, Eficacia y Cambio en Educación, 4(2e.).

Ceinos, C. (2004). Diagnóstico de las competencias de los orientadores laborales en el uso de las tecnologías de la información y de la comunicación. Santigo de Compostela: Universidad Santiago de Compostela. 
Chomsky, N. (1980). Rules and representations. The Behavioural and Brain Sciences, 3(1), 1-15. https:// doi.org/10.1017/S0140525X00001515

Collins, K. (2007). The concept of competence in the development of vocational education and training in selected EU member status : a critical analisis. Journal of Vocational Education \& Training, 67-88. https://doi.org/10.1080/13636820601145630

Dalziel, M. C. (1996). Las competencias: clave para una gestiónintegrada de los recursos humanos. Madrid: Deusto.

De la Orden Hoz, A. (2011). Reflexiones en torno a las competencias como objeto de evaluación en el ámbito educativo. Revista Electrónica de Investigación Educativa, 1-21. Recuperado de http://redie. uabc.mx/vol13no2/contenido-delaorden2.html

Delamare Le Deist, F. y Winterton, J. (2005). What is competence? Human Resource Development International, 8(1), 27-46. https://doi. org/10.1080/1367886042000338227

DeSeCo (2002). Definition and Selection of Competencies. Organización para la Cooperación y el Desarrollo Económico (OCDE). Recuperado de https://binomicos.wordpress.com/introduccion/ deseco/

D'Hainaut, L. (1980). La evaluación del contenido de un currículo. Programas de estudio de educación permanente. París: Unesco.

Díaz-Barriga, A. (2006). El enfoque de competencias en la educación. ¿Una alternativa o un disfraz de cambio? Perfiles Educativos, 28(111), 7-36.

Díaz-Barriga, A. (2010). Los profesores ante las innovaciones curriculares. Revista Iberoamericana de Educación Superior (Ries), 1(1), 37-57. https://doi. org/10.22201/iisue.20072872e.2010.1.15

Díaz-Barriga, A. (2011). Competencias en educación. Corrientes de pensamiento e implicaciones para el currículo y el trabajo en el aula. Revista Iberoamericana de Educación Superior (Ries), 2(5). https:// doi.org/10.22201/iisue.20072872e.2011.5.44

Eraut, M., (1994). Developing professional knowledge and competence. Londres: Falmer.

Fernández Guillermet, A. y Rubau, C. (2012). El enfoque por competencias en educación superior: conceptos claves, debates y aplicaciones en América Latina. XI Seminario Argentino-Chileno y $V$ Seminario Cono Sur de Ciencias Sociales, Humanidades y relaciones Internacionales. Cuyo: Instituto Balseiro (CNEA-UNCuyo), Instituto de Ciencias Básicas (UNCuyo).

Fernández Santamaría, M. (2000). Foro mundial sobre la educación calidad y equidad educativas. Revista Iberoamericana de Educación, 22, 193-197.

Ferreyra, H., Ariel, S., Peretti, G., Fontana, M., Pasut, M., Seara, R.,... Scheneider, C. (2008). De aprendizajes, competencias y capacidades en la educación primaria. Desandando caminos para construir nuevos senderos. Iberoamericana de Educación.

García San Pedro, M. J. (2010). Diseño y evaluación de un modelo de competencias en la universidad. Barcelona: UAB.

García Sanz, M. y Morillas Pedreño, L. (2011). La planificación de evaluación de competencias en educación superior. Revista Electrónica Interuniversitaria de Formación del Profesorado, 14(1), 113-124.

Garibay, J. (2011). Reforma educativa en Estados Unidos.

Gimeno Sacristan, J. (2001). Políticas y prácticas culturales en las escuelas: los abismos del a etapa posmodernapostmoderna. Revista Electrónica Heuresis, 2(1), 7-43.

Gimeno Sacristán, J. (2008). Educar por competencias, ¿qué hay de nuevo? Madrid: Morata.

Gimeno Sacristán, J. P. (2009). Educar por competencias, ¿qué hay de nuevo? Madrid.: Morata.

Gonczi, A. (1994). Developing a competent workforce. Adelaide: National Centre for Vocational Education Research.

Guerrero, A. (1999). El enfoque de las competencias profesionales: una solución conflictiva a la relación entre formación y empleo. Revista Complutense de Educación, 10(1), 335-360.

Hager, P. (2011). Recognition of informal learning: chaIlenges and issues. Journal of Vocational Education and Training, 521-535.

Irigoin, M. y Vargas, F. (2002). Competencia laboral. Manual de conceptos, métodos y aplicaciones. 
Montevideo: Cinterfor. Recuperado de https:// www.oitcinterfor.org/sites/default/files/file_publicacion/man_ops.pdf

Jonnaert, P. (2008). Logique de compétences et développement curriculaire. París: L'Harmattan.

Kemmis, S. (1993). El curriculum más allá de la teoría de la reproducción. Madrid: Morata.

Le Boterf, G. (2000). Ingeniería de las competencias. Barcelona: Gestión 2000, Epise.

Levy-Leboyer, C. (1997). Evaluación del personal. Madrid: Diaz de Santos Editores.

Mastache, A. (2009). Formar personas competentes. Desarrollo de competencias tecnológicas y psicosociales. Buenos Aires: Noveduc.

Mateo, J. (2007). Interpretando la realidad, construyendo nuevas formas de conocimiento: el desarrollo competencial y su evaluación. Revista de Investigación Educativa (RIE), 2(25), 513-531.

McClelland, D. (1973). Testing for Competencies rather than intelligence. American Psychologist, 1-14. https://doi.org/10.1037/h0034092

Ministerio de Educación Nacional (2005). Desarrollo por competencias.

Mertens, L. (1997). Competencia laboral: sistemas, surgimiento y modelo. Montevideo, Uruguay: Cinter-FOR/OIT.

Mertens, L. (1998). Desarrollo de un curriculum y sus variantes SCID y AMOD. OIT-Cintefort.

Mira Solves, J. J., Galán Vallejo, M., Van Kemenade, E., Marzo Campos, J. C., Blaya Salvador, I. y Pérez Jover, M. V. (enero-abril de 2012). Retos para el gobierno de las universidades en el marco del EEES. Revista de educación, 357, 445-465. DOI: https:// doi.org/10-4438/1988-592X-RE-2010-357-068

Mitrani, A., Dalziel, M. y Suárez de Puga, I. (1992). Las competencias. claves para una gestión integrada de los recursos humanos (Vol. 1). Bilbao: Ediciones Deusto S. A.

Mulder, M. (1989). New office technology: a study on curriculum design. Journal of European Industrial Training, 4(14), 19-28. https://doi.org/10.1108/ EUM0000000000194

Mulder, M. y Thijsen, A. (1990). Decision making in curriculum conferences : a study of convergence of opinion. Journal of Curriculum Studies, 22(4), 343360. https://doi.org/10.1080/0022027900220404

Nijhof, N. y Mulder, M. (1989). New office: technology: a study on curriculum design. Journal of European Industrial Training, 13(4), 19-28. https://doi. org/10.1108/EUM0000000000194

Norris, N. (1991). The trouble with competence. Cambridge Journal of Education, 21(3), 331-341. https://doi.org/10.1080/0305764910210307

OCDE (2012). Evaluaciones de políticas nacionales de educación. La educación superior en Colombia. Bogotá, Colombia: Banco Internacional de Reconstrucción y Fomento, Banco Mundial. http://dx.doi. org/10.1787/9789264180710-es

Pavié, A. (2011). Formación docente: hacia una definición del concepto de competencia profesional docente. Electrónica Interuniversitaria de Formación del Profesorado, 14 (1), 67-80.

Perrenoud, P. (2005). Diez nuevas competencias para enseñar. Educación Siglo XXI, 23-159.

Rodríguez Uribe, H. (2009). Pedagogía humanista. Fundamentos del currículo y calidad educativa. Pátzcuaro: U. I. Michoacán, Ed.

Rodríguez, V. (2004). Acerca de las competencias cognitivas. Revista Enfoques, 67-73.

Rychen, D. y Salganik, L. H. (2003). Keys competencies for a Successful Life and Well Functioning Society. Göttingen, Alemania: Hogrefe \& Huber Publishers.

Schwartz, S. (1995). Beyond individualism and collectivism: New cultural dimensions of values. En U. Kim, H.C. Triandis, C. Kagitcibasi, S-C. Choi y G. Yoon (eds.), Individualism and collectivism: Theory, method and application (pp. 85-119). Newbury Park, CA: Sage.

Spencer Jr., L. y Spencer, S. (1993). Evaluación de competencias en el trabajo. Nueva York: Wiley \& Sons.

Tardif, J. (2006). L'evaluation des compétences. Montreal: Chenelière Education.

Tierno García, J. I. (2011). El compromiso organizativo e institucional para diseñar y evaluar competencias en la universidad. Revista en Educación, 361.

Tobón, S. (2008). Formación basada en competencias. Bogotá: Escoe ediciones. 
Torres Santomé, J. (2008). Diversidad cultural y contenidos escolares. Revista de Educación, 345, 83-110.

Tuning, P. (2000-2001). Competencias proyecto Tuning-Europa, Tuning.-America Latina. nhbs/mtc. Recuperado de http://www.cca.org.mx/profesores/cursos/hmfbcp_ $\mathrm{ut} / \mathrm{pdfs} / \mathrm{m} 1 /$ competencias_proyectotuning.pdf

Unesco (2000). Informe final del Foro Mundial sobre la Educación Dakar. Recuperado de http://unesdoc. unesco.org/images/0012/001211/121117s.pdf

Vila Morales, D. (2011). Las concepciones de curriculum y su importancia en la elaboración de un doctorado curricular. Revista Cubana Estomato, 48(3), 145.
Voorhees, R. (2002). Competency-Based Learning Models: A Necessary Future. N. D. Research, Ed., 2001(110), 5-13. https://doi.org/10.1002/ir.7

Wesselink, R., Biemans, H. y Van den Elsen, E. (2005). Conceptual framework for competence-based. VET in the Netherlands. Recuperado de https://www. researchgate.net/publication/237705988_Developing_competence-based_VET_in_the_Netherlands Zayas Agüero, P. (mayo de 2010). Las competencias. Una visión teórico-metodológica. Contribuciones a la Economía. 